MODELING, IDENTIFICATION AND CONTROL, 1993, VOL. 14, NO. 4, 229-237

doi:10.4173/mic.1993.4.4

\title{
Adaptive feedback linearization applied to steering of ships
}

\author{
THOR I. FOSSEN† and MARIT J. PAULSEN $\dagger$
}

Keywords: Ship autopilot, ship steering, nonlinear control, adaptive control

This paper describes the application of feedback linearization to automatic steering of ships. The flexibility of the design procedure allows the autopilot to be optimized for both course-keeping and course-changing manoeuvres. Direct adaptive versions of both the course-keeping and turning controller are derived. The advantages of the adaptive controllers are improved performance and reduced fuel consumption. The application of nonlinear control theory also allows the designer in a systematic manner to compensate for nonlinearities in the control design.

\section{Introduction}

The reason for applying more sophisticated autopilots for ship steering are mainly due to fuel economy and performance improvement (Van Amerongen (1984)). Since the ship steering equations of motion are highly nonlinear and the model parameters depend on the forward speed as well as the weather conditions, nonlinear and adaptive control theory should be considered. A general approach based on adaptive feedback linearization can be used for this purpose. In fact, adaptive feedback linearization can be combined with optimal control theory to design an autopilot for course-keeping and fuel reduction. Similarly, pole-placement techniques can be applied to course-changing manoeuvres. This flexibility allows the designer to tune the autopilot for both coursekeeping and course-changing manoeuvres instead of designing two independent autopilots to accomplish these tasks.

The paper is organized as follows: Section 2 discusses state-of-the-art linear and nonlinear models for ship steering. In Section 3 the combined course-keeping and turning controller is presented. Section 4 contains a simulation study while Section 5 summarizes the conclusions.

\section{Ship steering equations of motion}

Some of the most common mathematical models describing the steering dynamics of ships are briefly reviewed in this section.

The models of Nomoto et al. (1957)

For small rudder angles, the transfer function between the rudder angle $\delta$ and the yawing rate $r$ of a surface ship can be described by the linear models of Nomoto et al. (1957). Nomoto's 2nd order model is written as

$$
\frac{r(s)}{\delta(s)}=\frac{K\left(1+T_{3} s\right)}{\left(1+T_{1} s\right)\left(1+T_{2} s\right)}
$$

Received 6 May 1993.

† Division of Engineering Cybernetics, The Norwegian Institute of Technology, N-7034 Trondheim, Norway.

(C) IEEE. Reprinted, with permission, from the Proceedings of the 1st IEEE Conference on Control Applications, Dayton, Ohio, 13-16 September 1992. 
where $s$ is used to denote the Laplace operator, $K$ is the gain constant and $T_{i}(i=1,2,3)$ are three time constants. A first-order approximation is obtained by defining the effective time constant as $T=T_{1}+T_{2}-T_{3}$. Hence,

$$
\frac{r(s)}{\delta(s)}=\frac{K}{1+T s}
$$

The yaw angle $\psi(t)$ is related to the yaw rate $r(t)$ as $\dot{\psi}(t)=r(t)$.

\section{The model of Bech and Wagner Smith (1969)}

The linear ship steering equations of motion can be modified to describe large rudder angles and course-unstable ships by simply adding a nonlinear manoeuvring characteristic to Nomoto's 2nd order model. Bech and Wagner Smith (1969) propose the model

$$
T_{1} T_{2} \psi^{(3)}+\left(T_{1}+T_{2}\right) \dot{\psi}+K H_{B}(\dot{\psi})=K\left(\delta+T_{3} \delta\right)
$$

where the function $H_{B}(\psi)$ describes the nonlinear manoeuvring characteristic produced by Bech's reverse spiral manoeuvre, that is

$$
H_{B}(\dot{\psi})=b_{3} \dot{\psi}^{3}+b_{2} \dot{\psi}^{2}+b_{1} \dot{\psi}+b_{0}
$$

For a course-unstable ship we will have that $b_{1}<0$ while a course-stable ship satisfies $b_{1}>0$. A single screw propeller or asymmetry in the hull will cause a non-zero value of $b_{0}$. Similarly, symmetry in the hull implies that $b_{2}=0$. Since a constant rudder angle is required to compensate for constant or slowly-varying wind and current disturbances, the bias term $b_{0}$ could conveniently be treated as an additional rudder off-set. This in turn implies that a large number of ships can be described by

$$
H_{B}(\dot{\psi})=b_{3} \dot{\psi}^{3}+b_{1} \dot{\psi}
$$

The model of Norrbin (1963)

Similarly, an extension of Nomoto's 1st order model can be made by defining

$$
T \ddot{\psi}+H_{N}(\dot{\psi})=K \delta
$$

where the manoeuvring characteristic $H_{N}(\dot{\psi})$ is defined as

$$
H_{N}(\dot{\psi})=n_{3} \dot{\psi}^{3}+n_{2} \dot{\psi}^{2}+n_{1} \dot{\psi}+n_{0}
$$

The Norrbin coefficients $n_{i}(i=0 \ldots 3)$ are related to those of Bech's model by $n_{i}=b_{i} /\left|b_{1}\right|$ $(i=0 \ldots 3)$. Hence, $n_{1}=-1$ for a course-unstable ship while $n_{1}=1$ corresponds to a course-stable ship.

\section{Forward speed effects}

The influence of the forward speed $U$ on the model parameters can be removed by using non-dimensional quantities. Let $L$ be the length of the hull. Hence, the gain and time constant can be made dimensionless with respect to speed variations by applying the transformations

$$
K=K^{*}(U / L) ; \quad T=T^{*}(L / U)
$$

where $K^{*}$ and $T^{*}$ are dimensionless constants, typically in the range of $0.5-2.5$ for most ships (Van Amerongen (1984)). 


\section{Feedback linearization}

The basic idea with feedback linearization is to transform the nonlinear systems dynamics into a linear system (Freund (1973)). Conventional control techniques like pole placement and linear quadratic optimal control theory can then be applied to the linear system. In robotics, this technique is commonly referred to as computed torque control. Adaptive computed torque control have been applied to robot manipulators by Horowitz and Tomizuka (1986) and to underwater vehicles by Fossen (1991). Consider Norrbin's nonlinear ship steering equations of motion in the form

$$
m \dot{\psi}+d_{1} \dot{\psi}+d_{3} \dot{\psi}^{3}=\delta
$$

where $m=T / K, d_{1}=n_{1} / K$ and $d_{3}=n_{3} / K$. For simplicity, the coefficients $n_{0}$ and $n_{2}$ are assumed to be zero. Taking the control law to be

$$
\delta=\hat{m} a_{\psi}+\hat{d}_{1} \dot{\psi}+\hat{d}_{3} \dot{\psi}^{3}
$$

where the hat denotes the estimates of the parameters and $a_{w}$ can be interpreted as the commanded acceleration, yields

$$
m\left(\dot{\psi}-a_{\psi}\right)=\tilde{m} a_{\psi}+\tilde{d}_{1} \dot{\psi}+\tilde{d}_{3} \dot{\psi}^{3}
$$

Here $\tilde{m}=\hat{m}-m, d_{1}=\hat{d}_{1}-d_{1}$ and $\tilde{d}_{3}=\hat{d}_{3}-d_{3}$ are the parameter errors. Consequently, the error dynamics can be made globally asymptotically stable by proper choices of the commanded acceleration $a_{\psi}$. The unknown parameters $m, d_{1}$ and $d_{3}$ are made dimensionless with respect to speed variations by applying the transformations

$$
m=m^{*}(L / U)^{2} ; \quad d_{1}=d_{1}^{*}(L / U) ; \quad d_{3}=d_{3}^{*}(L / U)^{3}
$$

where the asterisk denotes the non-dimensional quantities (with respect to speed $U$ and length of hull $L$ ). Hence, the error equation (11) can be expressed as

$$
m^{*}\left(\dot{\psi}-a_{\psi}\right)=\tilde{m}^{*} a_{\psi}+\tilde{d}_{1}^{*}(U / L) \dot{\psi}+\tilde{d}_{3}^{*}(L / U) \dot{\psi}^{3}
$$

The control law (10) can be made adaptive by including a parameter estimator for updating of $\hat{m}, \tilde{d}_{1}$ and $\tilde{d}_{3}$. We will discuss two methods utilizing pole-placement and optimal control theory, respectively.

\subsection{Pole-placement Course-changing}

In the case of no parametric uncertainties, equation (13) reduces to

$$
\ddot{\psi}=a_{\psi}
$$

which suggests that the commanded acceleration should be chosen as

$$
a_{\psi}=\ddot{\psi}_{d}-K_{d} \tilde{\psi}-K_{p} \tilde{\psi}
$$

where $\psi_{d}$ is the desired heading angle and $\tilde{\psi}=\psi-\psi_{d}$ is the heading error. This in turn yields the error dynamics

$$
\ddot{\psi}+K_{d} \tilde{\psi}+K_{p} \tilde{\psi}=0
$$

The desired states: $\psi_{d}, \psi_{d}$ and $\psi_{d}$ can be generated by the reference model shown in Fig. 1 where $\psi_{r}$ is the commanded input. 


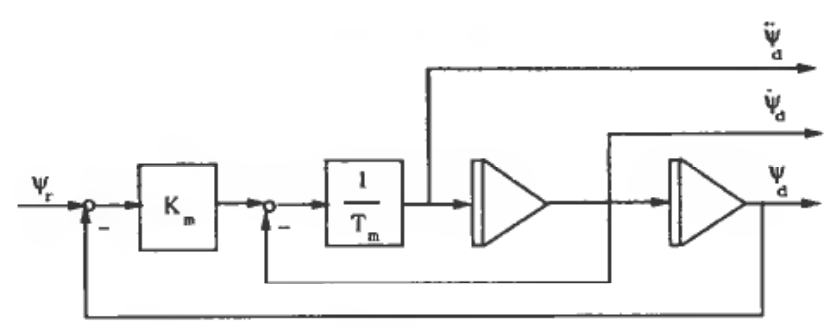

Figure 1. Reference model for turning controller.

A simple pole-placement algorithm could be to choose:

$$
K_{p}=\lambda^{2} ; \quad K_{d}=2 \lambda ; \quad \lambda>0
$$

which yields the critically damped error dynamics:

$$
\ddot{\tilde{\psi}}+2 \lambda \tilde{\psi}+\lambda^{2} \tilde{\psi}=0
$$

or equivalently,

$$
\dot{s}+\lambda s=0 \quad \text { with } \quad s=\tilde{\psi}+\lambda \tilde{\psi}
$$

Here $s$ can be interpreted as a measure of tracking. An adaptive version of the poleplacement algorithm is given in Theorem 3.1.

\section{Theorem 3.1. (Adaptive pole-placement)}

The parameter update laws

$$
\begin{aligned}
& \hat{m}^{*}=-\gamma_{1} a_{\psi} s \\
& \dot{\hat{d}}_{1}^{*}=-\gamma_{2}(U / L) \dot{\psi} s \\
& \dot{\hat{d}}_{3}^{*}=-\gamma_{3}(L / U) \dot{\psi}^{3} s
\end{aligned}
$$

with $\gamma_{\mathrm{i}}>0$ for $(i=1,2,3)$ yields a globally stable system.

Proof: Substituting equations (15) and (17) into the error dynamics (13), yields

$$
m^{*}(\dot{s}+\lambda s)=\tilde{m}^{*} a_{\psi}+\tilde{d}_{1}^{*}(U / L) \dot{\psi}+\mathcal{d}_{3}^{*}(L / U) \psi^{3}
$$

Consider the Lyapunov function candidate

$$
V\left(s, \tilde{m}^{*}, \tilde{d}_{1}^{*}, \tilde{d}_{3}^{*}\right)=\frac{1}{2}\left[m^{*} s^{2}+\frac{1}{\gamma_{1}}\left(\tilde{m}^{*}\right)^{2}+\frac{1}{\gamma^{2}}\left(\tilde{d}_{1}^{*}\right)^{2}+\frac{1}{\gamma^{3}}\left(\tilde{d}_{3}^{*}\right)^{2}\right]
$$

Consequently, differentiation of $V$ with respect to time together with (21), yields

$$
\dot{V}=-\lambda m^{*} s^{2}+\tilde{m}^{*}\left[\frac{1}{\gamma_{1}} \dot{\tilde{m}^{*}}+a_{\psi} s\right]+\tilde{d}_{1}^{*}\left[\frac{1}{\gamma^{2}} \tilde{d}_{1}^{*}+\frac{U}{L} \dot{\psi} s\right]+\tilde{d}_{3}^{*}\left[\frac{1}{\gamma^{3}} \hat{f}_{3}^{*}+\frac{L}{U} \dot{\psi}^{3} s\right]
$$

Assuming that $\dot{m}^{*}=\dot{d}_{1}^{*}=\dot{d}_{\mathbf{3}}^{*}=0$, the particular choice (20) of the parameter update laws implies that (23) reduces to

$$
\dot{V}=-\lambda m^{*} s^{2} \leqslant 0
$$

This implies that $V(t) \leqslant V(0)$, and therefore that $s, \tilde{m}^{*}, \partial_{1}^{*}$ and $\tilde{d}_{3}^{*}$ remains bounded in all time. Differentiating $V$ with respect to time, yields

$$
\ddot{V}=-2 \lambda m^{*} s \dot{s}
$$


Assuming that $a_{w}$ is bounded implies that $\sin (21)$ is bounded and consequently that $\ddot{V}$ is bounded. Hence, $\dot{V}$ must be uniform continuous. Convergence of $\dot{V}$ to zero is guaranteed by application of Barbălat's lemma (see Appendix A). This in turn implies that the $s \rightarrow 0$ as $t \rightarrow \infty$. In view of $s=\tilde{\psi}+\lambda \tilde{\psi}$, this implies that the tracking error $\tilde{\psi}$ converges asymptotically to zero. Notice that, convergence of the parameter errors $\tilde{m}^{*}$, $\tilde{d}_{1}^{*}$ and $\tilde{d}_{3}^{*}$ requires that the system is persistently exciting (PE).

\section{Integral action}

In the implementation of the control law it is desirable to include integral action to ensure that the tracking error converges to zero in the presence of constant wind and current disturbances. This can be done by simply modifying the measure of tracking according to

$$
s=\tilde{\psi}+2 \lambda \tilde{\psi}+\lambda^{2} \int_{0}^{t} \tilde{\psi}(\tau) d \tau
$$

which suggests that the commanded acceleration should be chosen as

$$
a_{\psi}=\ddot{\psi}_{d}-3 \lambda \tilde{\psi}-3 \lambda^{2} \tilde{\psi}-\lambda^{3} \int_{0}^{t} \tilde{\psi}(\tau) d \tau
$$

\subsection{Linear quadratic optimal control course-keeping}

During course-keeping it is desirable to minimize an energy cost function relating the control energy to the fuel consumption of the ship. Since the term proportional to $\dot{\psi}^{3}$ is small during course-keeping, we will restrict our analysis to the Nomoto model

$$
m \dot{\psi}+d \dot{\psi}=\delta
$$

Let $\psi_{r}=$ constant denote the desired heading. Hence, an optimal course-keeping controller can be derived for the 2 nd order system

$$
\ddot{\psi}=a_{\psi}
$$

by minimizing the quadratic cost function

$$
J=\frac{1}{2} \int_{0}^{\mathrm{T}}\left[\left(\psi_{\mathrm{r}}-\psi\right)^{2}+\lambda_{1} \psi^{2}+\lambda_{2} a_{\psi}^{2}\right] d \tau
$$

weighting the tracking error $\psi_{r}-\psi$ against the yawing rate $\psi$ and the commanded acceleration $a_{\psi}$ with weighting factors $\lambda_{1}$ and $\lambda_{2}$, respectively. This yields the following steady-state solution for the optimal commanded acceleration

$$
a_{\psi}=K_{p}\left(\psi_{r}-\psi\right)-K_{d} \dot{\psi}
$$

with

$$
K_{p}=\left(\frac{1}{\lambda_{2}}\right)^{1 / 2} ; \quad K_{d}=\left(2\left(\frac{1}{\lambda_{2}}\right)^{1 / 2}+\frac{\lambda_{1}}{\lambda_{2}}\right)^{1 / 2}
$$

Integral action may be obtained by modifying the commanded acceleration according to

$$
a_{w}=K_{p}\left(\psi_{r}-\psi\right)-K_{d} \psi+K_{i} \int_{0}^{\mathrm{T}}\left[\psi_{r}-\psi(\tau)\right] d \tau
$$


where a suitable choice of $K_{i}$ is

$$
K_{i}=\frac{K_{p}}{5 K_{d}}
$$

This corresponds to $T_{i}=5 T_{d}$ in a PID controller. The control input is obtained by transforming the commanded acceleration according to

$$
\delta=\hat{m} a_{\psi}+\hat{d} \dot{\psi}
$$

where the hat denotes the parameter estimates. Consequently, the error dynamics is obtained as

$$
m^{*}\left(\ddot{\psi}-a_{\psi}\right)=\tilde{m}^{*} a_{\psi}+\tilde{d}^{*}(U / L) \dot{\psi}
$$

Optimality with respect to (30) require that $\tilde{m}^{*}=\tilde{d}^{*}=0$ (no parametric uncertainties). This suggests that adaptive control theory should be applied. Let the error dynamics (36) be written as

where $x=\left(\psi-\psi_{r}, \psi\right)^{\mathrm{T}}$ and

$$
\dot{\boldsymbol{x}}=\boldsymbol{A} \boldsymbol{x}+\boldsymbol{b} \frac{1}{m^{*}} \boldsymbol{\phi}^{\mathrm{T}} \tilde{\tilde{\theta}^{*}}
$$

$$
\begin{aligned}
& \boldsymbol{A}=\left[\begin{array}{cc}
0 & 1 \\
-K_{p} & -K_{d}
\end{array}\right] \quad \boldsymbol{b}=\left[\begin{array}{l}
0 \\
1
\end{array}\right] \\
& \boldsymbol{\phi}=\left[a_{\psi}, \frac{U}{L} \psi,\right]^{\mathrm{T}} \quad \tilde{\theta}^{*}=\left[\tilde{m}^{*}, \tilde{d}^{*}\right]^{\mathrm{T}}
\end{aligned}
$$

Theorem 3.2 (Adaptive linear quadratic optimal control)

The control law (10) with the optimal commanded acceleration (31) and the parameter update law

where $e=\boldsymbol{c}^{\mathrm{T}} \boldsymbol{x}$ and

$$
\dot{\hat{\theta}}^{*}=-\boldsymbol{\Gamma}^{-1} \phi e ; \quad \boldsymbol{\Gamma}=\boldsymbol{\Gamma}^{\mathrm{T}}>0
$$

$$
\begin{aligned}
\boldsymbol{A}^{\mathrm{T}} \boldsymbol{P}+\boldsymbol{P A} & =-\boldsymbol{Q} \\
\boldsymbol{P} \boldsymbol{b} & =\boldsymbol{c}
\end{aligned}
$$

and $\boldsymbol{P}=\boldsymbol{P}^{\mathrm{T}}>0$ and $\boldsymbol{Q}=\boldsymbol{Q}^{\mathrm{T}}>0$, yields a globally stable system.

Proc f: Define a scalar Lyapunov function

$$
V\left(\mathbf{x}, \dot{\theta}^{*}\right)=\mathbf{x}^{\mathrm{T}} \boldsymbol{P} \mathbf{x}+\frac{1}{m^{*}} \tilde{\boldsymbol{\theta}}^{* \mathrm{~T}} \mathbf{\Gamma} \tilde{\boldsymbol{\theta}}^{*}
$$

Differentiating $V$ with respect to time together with (37), yields

$$
\dot{\boldsymbol{V}}=\boldsymbol{x}^{\mathrm{T}}\left(\boldsymbol{A}^{\mathrm{T}} \boldsymbol{P}+\boldsymbol{P A}\right) \boldsymbol{x}+\frac{2}{m^{*}}\left(\dot{\boldsymbol{\theta}}^{\mathrm{T}}\left(\boldsymbol{\Gamma} \dot{\boldsymbol{\theta}}^{*}+\phi \boldsymbol{b}^{\mathrm{T}} \boldsymbol{P} \mathbf{x}\right)\right.
$$

Finally, substituting of equations (39) and (40) into (42) yields

$$
\dot{V}=-\mathbf{x}^{\mathrm{T}} \boldsymbol{Q} \mathbf{x} \leqslant 0
$$

which according to Lyapunov stability theory for autonomous systems ensures that $\psi(t) \rightarrow \psi_{\mathrm{r}}$ as $t \rightarrow \infty$ and that $\tilde{\theta}^{*}(t)$ is bounded. $\tilde{\theta}^{*}(t)$ will converge to zero if the system is PE. 


\section{Simulation study}

The ship model used in the simulation study is adapted from Van Amerongen (1982). In this reference, 'the R.O.V. Zeefakkel' is described by the following set of parameters

$$
\begin{aligned}
L & =45(\mathrm{~m}) & U & =5 \cdot 0(\mathrm{~m} / \mathrm{s}) \\
K & =0.5\left(\frac{1}{\mathrm{~s}}\right) & b_{1} & =2 \cdot 0(-) \\
T & =31(\mathrm{~s}) & b_{3} & =0.8\left(\mathrm{~s}^{2}\right)
\end{aligned}
$$

where $b_{1}$ and $b_{3}$ are found from the reversed spiral test. Consequently,

$$
n_{1}=b_{1} /\left|b_{1}\right|=1 \cdot 0 ; \quad n_{3}=b_{3} /\left|b_{1}\right|=0 \cdot 4\left(\mathrm{~s}^{2}\right)
$$

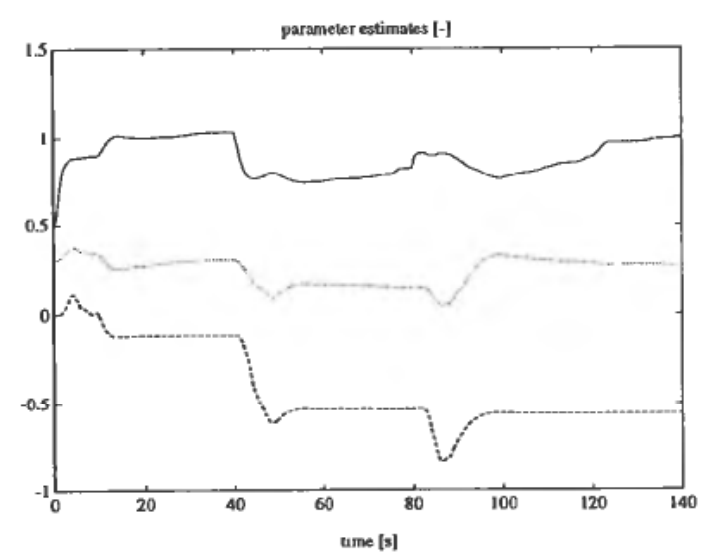

Figure 2. Parameter estimated, $\hat{m}^{*}$ (solid line), $\hat{d}_{1}^{*}$ (points) and $\hat{d}_{3}^{*}$ (dashed line) versus time (not PE).
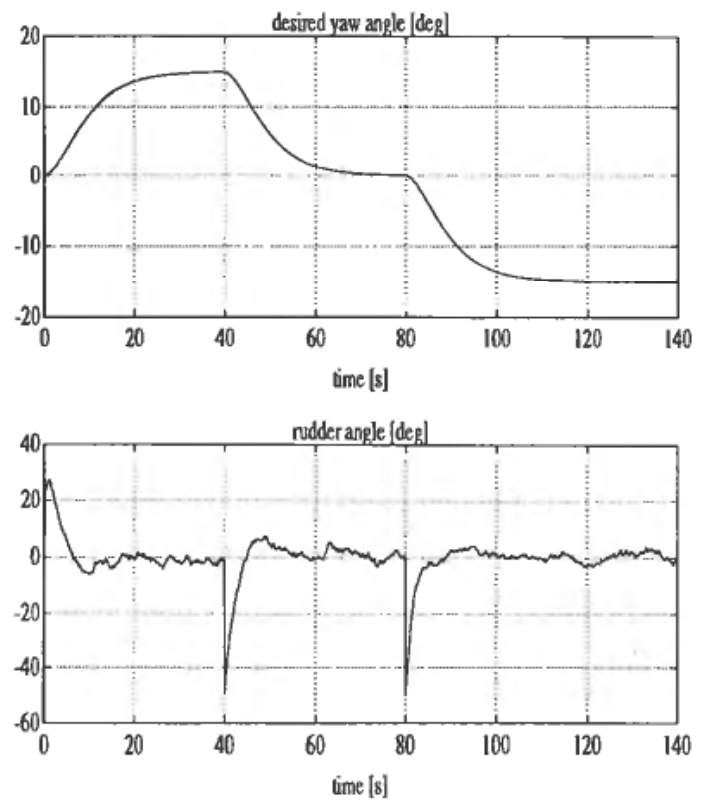

Figure 3. Desired yaw angle $\psi_{d}$ and rudder angle $\delta$ versus time. 

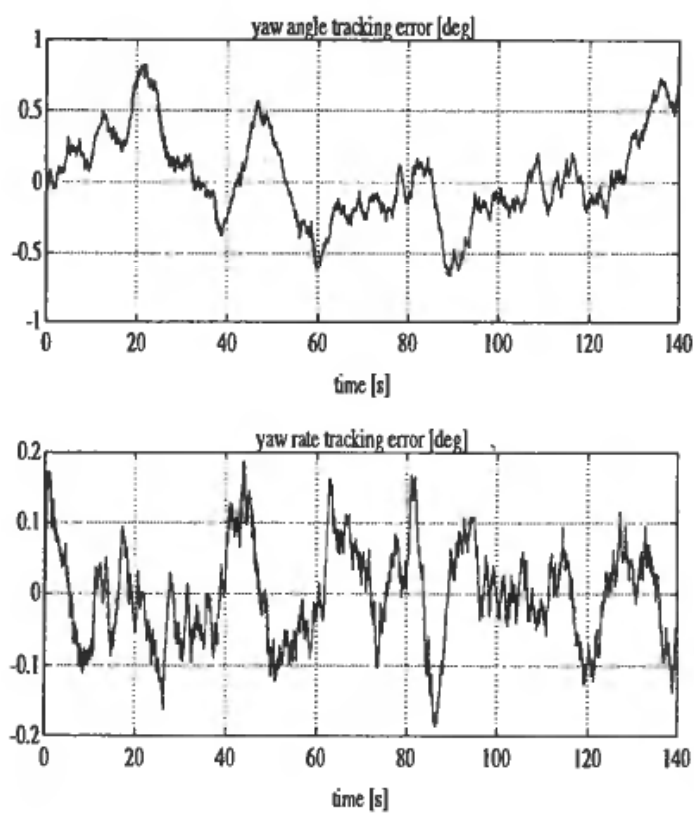

Figure 4. Yaw angle tracking error $\tilde{\psi}$ and yaw rate tracking error $\dot{\tilde{\psi}}$ versus time.

which clearly is a course-stable ship. In the simulation study a sampling rate of $10 \mathrm{~Hz}$ was used. The controller parameters were chosen as (Theorem 3.1) $\gamma_{1}=10^{4}, \gamma_{2}=10^{4}$, $\gamma_{3}=10^{6}$ and $\lambda=0 \cdot 2$. The measurement noise was chosen as Gaussian white noise processes with maximum amplitude of $0.1(\mathrm{deg})$ and $0.05(\mathrm{deg} / \mathrm{s})$ for the yaw angle and the yaw rate, respectively. The parameter estimates: $\hat{m}^{*}, \hat{d}_{1}^{*}$ and $\hat{d}_{3}^{*}$ during coursechanging are shown in Fig. 2.

The desired yaw angle $\psi_{d}$ and the rudder angle $\delta$ is shown in Fig. 3 while the yaw angle and yaw rate tracking errors are shown in Fig. 4.

\section{Conclusions}

A direct adaptive autopilot based on feedback linearization has been derived. Particular attention has been made towards course-keeping and course-changing manoeuvres. It is believed that the optimal course-keeping autopilot will be superior conventional autopilots with respect to performance and fuel economy. The performance of the turning controller was illustrated through a simulation study. Global stability of both adaptive controllers was proven by applying nonlinear stability theory.

\section{ACKNOWLEDGMENTS}

This work was partially sponsored by Robertson Tritech A.S. in Egersund, Norway and the Royal Norwegian Council for Scientific and Industrial Research.

\section{Appendix A. Stability theory}

During tracking of time-varying reference trajectories like course-changing manoeuvres the following lemmas from non-autonomous stability theory can be applied (Popov (1973)). 
Lemma A.I (Barbălat's lemma)

If the function $g(t)$ has a finite limit as $t \rightarrow \infty$, is differentiable and $\dot{g}(t)$ is uniformly continuous, then $\dot{g}(t) \rightarrow 0$ as $t \rightarrow \infty$. The proof is found in Barbălat (1959).

Lemma A.2 (Lemma for global stability)

Assume that there exists a scalar function $V(x, t)$ satisfying

- $V(x, t)$ is lower bounded

- $\dot{V}(x, t)$ is negative semi-definite

- $\dot{V}(x, t)$ is uniformly continuous in time

then $\dot{V}(x, t) \rightarrow 0$ as $t \rightarrow \infty$.

Remark: (Uniform continuity)

A sufficient condition for a differentiable function $\dot{V}(x, t)$ to be uniformly continuous is that its derivative $\ddot{V}(x, t)$ is bounded $\forall t \geqslant t_{0}$.

\section{REFERENCES}

BARBǍLAT, (1959). Systèmes d'equations diffèrentielles d'oscillations non linéaires. Revue de Mathèmatiques Pures et Appliquées, 4, 267-270. (in French).

BECH, M. I. and WAGNER SMITH L. (1969). Analogue Simulation of Ship Manoeuvres. Technical Report Hy-14, Hydro- and Aerodynamics Laboratory, Lyngby, Denmark.

Fossen, T. I. (1991). Nonlinear Modelling and Control of Underwater Vehicles. Dring thesis, Division of Engineering Cybernetics, Norwegian Institute of Technology, Trondheim, June 1991.

FREUND, E., (1973). Decoupling and pole assignment in nonlinear systems. Electronics Letter, 9 , No. 16, 1973.

Horowitz, R. and Tomizuka, M., (1986). An adaptive control scheme for mechanical manipulators - compensation of nonlinearity and decoupling control. Technical Report No. 80-WA/DSC-6, ASME.

Nomoto, K., TAGUCHI, T., Honda, K. and Hirano, S. (1957). On the steering qualities of ships. Technical Report, International Shipbuilding Progress, Vo. 4.

Norrbin, N. H. (1963). On the design and analyses of the zig-zag test on base of quasi linear frequency response. Technical Report B 104-3, The Swedish State Shipbuilding Experimental Tank (SSPA), Gothenburg, Sweden.

PoPOV, V. M. (1973). Hyperstability of Control Systems (Springer-Verlag, Berlin.)

VAN AMERONGEN, J. (1982). Adaptive steering of ships - a model reference approach to improved manoeuvring and economical course keeping. Ph.D thesis, Delft University of Technology, The Netherlands.

VAN AMERONGEN, J. (1984). Adaptive steering of ships-a model reference approach. Automatica, 20, 3-14. 